\title{
Designer hydrophilic regions regulate droplet shape for controlled surface patterning and 3D microgel synthesis
}

\author{
Dr. Matthew J. Hancock ${ }^{\dagger}$, \\ Center for Biomedical Engineering, Department of Medicine, Brigham and Women's Hospital, \\ Harvard Medical School, 65 Landsdowne Street, Cambridge, MA 02139 (USA) \\ Dr. Fumiki Yanagawa ${ }^{\dagger}$, \\ Center for Biomedical Engineering, Department of Medicine, Brigham and Women's Hospital, \\ Harvard Medical School, 65 Landsdowne Street, Cambridge, MA 02139 (USA)

\section{Dr. Yun-Ho Jang,} \\ Center for Biomedical Engineering, Department of Medicine, Brigham and Women's Hospital, \\ Harvard Medical School, 65 Landsdowne Street, Cambridge, MA 02139 (USA) \\ Dr. Jiankang He, \\ Center for Biomedical Engineering, Department of Medicine, Brigham and Women's Hospital, \\ Harvard Medical School, 65 Landsdowne Street, Cambridge, MA 02139 (USA). State Key \\ Laboratory of Manufacturing Systems Engineering, Xi'an Jiaotong University, Xi'an, Shaanxi \\ 710049 (China)
}

\section{Dr. Nezamoddin N. Kachouie,}

Center for Biomedical Engineering, Department of Medicine, Brigham and Women's Hospital, Harvard Medical School, 65 Landsdowne Street, Cambridge, MA 02139 (USA)

Prof. Hirokazu Kaji, and

Center for Biomedical Engineering, Department of Medicine, Brigham and Women's Hospital, Harvard Medical School, 65 Landsdowne Street, Cambridge, MA 02139 (USA). Department of Bioengineering and Robotics, Graduate School of Engineering, Tohoku University, Sendai 980-8579 (Japan)

\section{Prof. Ali Khademhosseini}

Center for Biomedical Engineering, Department of Medicine, Brigham and Women's Hospital, Harvard Medical School, 65 Landsdowne Street, Cambridge, MA 02139 (USA). Partners Research Building, Room 252, 65 Landsdowne Street, Cambridge, MA 02139 (USA). Wyss Institute for Biologically Inspired Engineering, Harvard University, Boston, MA 02115 (USA). Harvard-MIT Division of Health Sciences and Technology, Massachusetts Institute of Technology, Cambridge, MA 02139 (USA)

Ali Khademhosseini: alik@rics.bwh.harvard.edu

\section{Keywords}

microdroplets; surface tension; surface patterning; microwell; cells; microgels

\footnotetext{
Correspondence to: Ali Khademhosseini, alikerics.bwh. harvard.edu.

$\dagger$ Authors contributed equally.

Supporting Information is available on the WWW under http://www.small-journal.com or from the author
} 
We present simple techniques for controlling the shapes of micro- and nanodrops on surfaces with special hydrophilic regions surrounded by hydrophobic boundaries. Finite element method simulations linked the shape of the hydrophilic regions to that of the droplets. We used shaped droplets to controllably pattern planar surfaces and microwell arrays with microparticles and cells at the micro- and macroscales. Droplets containing suspended sedimenting particles, initially at uniform concentration, deposited more particles under deeper regions than under shallow. The resulting surface concentration was thus proportional to the local fluid depth and agreed well with the measured and simulated droplet profiles. We also highlight a second application in which shaped droplets of prepolymer solution were crosslinked to synthesize microgels with tailored 3D geometry.

\section{Introduction}

Surfaces patterned with wetting and non-wetting regions can manipulate fluid morphology ${ }^{[1]}$ and direct flow, ${ }^{[2,3]}$ all without geometrical boundaries like channel walls. ${ }^{[4]}$ Computational tools to calculate droplet morphology are well established, ${ }^{[5,6]}$ providing key tools to design custom wetting/non-wetting regions to obtain the desired droplet shape.

The ability to precisely control droplet morphology has an array of engineering applications. Droplets have been used to direct the assembly of vertically aligned nanorods ${ }^{[7]}$ and to deposit micro-/nanoparticles in various structures on chemically and topographically templated surfaces. ${ }^{[8-10,11-13]}$ Custom wetting/non-wetting patterns have been used to control the morphology of glycerol on a surface for liquid molding applications. ${ }^{[14,15]}$ To date, few studies have leveraged the three-dimensional (3D) shapes of droplets to enhance these and other patterning techniques. ${ }^{[8,13-15]}$

The need to controllably pattern surfaces with microparticles and cells is also significant. ${ }^{[10,16,17]}$ To date, despite achieving complex surface patterns with particles or self-assembled particle aggregates, these have almost always been patterned at a single concentration. Linear gradients in particle surface concentration have been created by dipcoating/sintering ${ }^{[18]}$ and convection. ${ }^{[3,19,20]}$ Surfaces with gradients in particle affinity have been also prepared, such as those with gradients in cell attachment. ${ }^{[21]}$ Controlled evaporation through templates ${ }^{[8]}$ and in confined spaces ${ }^{[13]}$ provides additional control over particle patterning. Refined patterning fidelity could offer improvements to an array of engineering applications. ${ }^{[17,22]}$ The spatially varying surface morphology inherent in micro-/nanoparticle gradients can regulate cell spreading, proliferation, and actin formation. ${ }^{[23]}$ Chemoattractant microgradients near degradeable microspheres can induce cell migration toward the microspheres. ${ }^{[24]}$ Spatial gradients in cell density are also potentially useful for generating biomimetic tissue constructs (e.g., cartilage tissue). ${ }^{[25]}$ The shape and density of roughness elements on a surface can also regulate surface wetting properties. ${ }^{[6,26]}$

In this work, we present the design tools and benchtop fabrication methods for patterning surfaces with custom wetting/non-wetting regions to regulate 3D droplet shapes at the macro- and microscales. A range of droplet shapes were produced to validate our combined experimental and theoretical investigation. We highlighted the utility of shaped droplets by using them to pattern surfaces with microparticles and cells with controlled microscale spatial variations in surface concentration, such as sine waves, linear gradients and spiral gradients. Also, microwell arrays were seeded with predefined and spatially varying seeding densities. Finally, we engineered shaped hydrogels at the macro- and microscale with controlled 3D topography. 


\section{Results and discussion}

\subsection{Shaped droplets on surfaces patterned with hydrophilic and hydrophobic regions}

The shape of a droplet resting on a surface depends on its volume, the surface tension of the fluid, the contact angle, and the shape of the contact line. The shape of the contact line may be controlled by patterning the substrate with hydrophilic regions surrounded by hydrophobic boundaries. Finite element method (FEM) simulations allow surface coatings to be custom designed to obtain specific droplet shapes (Fig. 1a). Various benchtop methods exist to create surfaces with custom hydrophilic and hydrophobic patterns. At the macroscale, we employed three methods including "mask and spray",[20] "coated cutouts", and microcontact printing ${ }^{[27,28]}$ (Fig. 1b). In the mask and spray method, a precision-cut vinyl mask (Figs. S1 and S2) was fixed to a glass slide, both of which were then coated with hydrophobic spray. Once dry, the mask was removed, exposing the hydrophilic region. In the coated cutout method, the same precision-cut vinyl sheets (Figs. S1 and S2) were first coated with hydrophobic spray and allowed to dry. The region exterior to the shape (here called the "cutout") was peeled from the sheet and fixed to a glass slide (Fig. 1b). The microcontact printing method employs PDMS stamps with depressions in the shape of the hydrophilic patterns (Fig. 1b). The stamps were spin-coated with hydrophobic octadecyltrichlorosilane (OTS) solution and gently pressed onto glass slides. All three methods were used to pattern glass slides at the macroscale; at the microscale, only microcontact printing was used.

At the macroscale, fluid was pipetted onto the hydrophilic region and immediately conformed to its shape, yielding three-dimensional (3D) sine wave, wedge, staircase, spiral and square wave droplets (Figs. 1c, S4a-c). Similar shapes were observed on slides patterned with the mask and spray and coated cutout methods (Fig. 1c). At the microscale, a 5-10 $\mu \mathrm{L}$ droplet containing $0.5 \%$ Tween 20 surfactant was deposited on an OTS-patterned glass slide. The slide was housed in a humid Petri dish, which was tilted and tapped gently (Fig. S3a) to move the drop along the slide surface, leaving small microdroplets on each hydrophilic region (Figs. 1d, S4d,e). A host of microdroplet shapes were produced, including wedges, sine waves, square waves and spirals. These microdroplets conformed to the hydrophobic boundaries of the hydrophilic regions. Breath figures, ${ }^{[29]}$ tiny droplets that condensed on the hydrophobic region, were also observed.

To probe the effects of fluid volume, surface tension, boundary geometry and the coating method on droplet shape, we captured eye-level photographs of droplets and extracted the centerline depth profiles (Fig. 2). The depths of sine wave, wedge and staircase drops increased proportionally to fluid volume (Fig. 2a-c). Droplet depth increased with the wavelength of the sine pattern (at constant amplitude, Fig. 2a), and increased proportionally with the cross-sectional width of the wedge pattern (Fig. 2b). The profiles of staircase drops were characterized by plateaus joined by smooth transition regions (Fig. 2c). In all cases, similar drop profiles were obtained on slides coated with the mask and spray method and the coated cutout method (Fig. 2d). Tween 20 surfactant was added to water at a concentration of $0.01 \%$ (above the critical micelle concentration (CMC), $0.005 \%$ or $0.04 \mathrm{mM}$ ) to decrease the surface tension from 72 to 33 dynes $\mathrm{cm}^{-1},{ }^{[30]}$ but had little effect on the macrodroplet profiles (Fig. 2e). An additional test of the wavelength effect on drop morphology was performed with a multi-wedge region consisting of eight back-to-back wedges of the same maximum width $(4 \mathrm{~mm})$ but different lengths. Like the sine wave pattern, the droplet was deeper over the longer wedges.

To assess the ability to design hydrophilic/hydrophobic patterns to control droplet shape, we compared measured droplet profiles with FEM simulations performed in Surface Evolver. ${ }^{[31]}$ In the simulations, all spatial dimensions were scaled by the capillary length, $l_{c}$ 
$=(\sigma / \rho g)^{1 / 2}$, where $\sigma$ is the surface tension of the fluid/air interface, $\rho$ is the fluid density, and $\mathrm{g}$ is the acceleration of gravity. In our experiments, $l_{c}=2.7 \mathrm{~mm}$ for distilled water at $20{ }^{\circ} \mathrm{C}$ and $l_{c}=1.84 \mathrm{~mm}$ for distilled water plus Tween 20 above the CMC. Inputs to the simulations included the geometry of the hydrophilic region, in spatial coordinates scaled by the capillary length $l_{c}$, and the droplet volume scaled by $l_{c}^{3}$ (for details and computational code, see the Experimental section and SI Methods II). In all cases, simulations agreed well with measurements. Minor discrepancies were due to evaporation, minor variations in the coating pattern and, for macroscale droplets, minor variations in the spray coating thickness. An additional detail of note for macroscale drops was that the hydrophobic boundaries associated with the mask and spray and coated cutout methods had nonzero thickness, on average $57 \mu \mathrm{m}$ for the mask and spray method and $144 \mu \mathrm{m}$ for the coated cutout method (included vinyl plus coating; staircase coated cutout boundary had a total height of $125 \mu \mathrm{m}$ due to use of a different vinyl). Since the fluid volumes were such that droplets extended well above the coatings, for simplicity, only the portion of the droplet above the surface of the coating was modeled (for details, see SI Methods II).

The effect of scale was tested by measuring microdroplets of water on micropatterned hydrophilic regions (Fig. $2 \mathrm{~g}-\mathrm{i}$ ). To ensure full wetting of the microscale hydrophilic regions, $0.5 \%$ of the surfactant Tween 20 was added to the droplet solution. For visualization, $0.1 \%$ fluorescein isothiocyanate (FITC) was also added. For the small droplet volumes $(\sim \mathrm{nL})$, with depths of order $100 \mu \mathrm{m}$ or less, the relative intensity was proportional to the droplet depth. ${ }^{[14]}$ The centerline relative intensities measured for different droplets on the same pattern were superposed and compared well with FEM simulations of the centerline relative depth profiles of microdroplets of typical $\mathrm{nL}$ volumes (see SI Methods II for simulation details). The range of volumes for a particular microdroplet was estimated as that of the corresponding macrodroplet times the cube of the scale factor (1/10 in most cases) between the micro- and macroscales. Due to our simple benchtop wetting method, the microdroplet volumes were not accurately controlled in our microscale experiments. The simulated relative depth profiles varied somewhat for spiral droplets over the volume range $10-30 \mathrm{~nL}$, and hence variations in droplet volume were likely the reason for the observed variations in the measured relative centerline intensity profiles (Fig. 2g). The measured intensity profiles for the micro wedge and micro double sine wave patterns were quite close, and agreed well with FEM simulations based on typical droplet volumes (Fig. 2h,i).

\subsection{Patterning a surface with microparticles and cells using shaped droplets}

The shaped droplet platform offers a simple method of patterning microparticles and cells on surfaces with controlled 2D spatial variations in surface concentration. When the droplet solution contains sedimenting particles, initially at a uniform concentration, more particles are deposited under deep portions of the drop than under shallow portions. Thus, once the particles have all settled to the substrate under the force of gravity, the resulting concentration of particles on the substrate mimics the depth profile of the droplet (Fig. 3a). Figure $3 \mathrm{~b}$ shows the deposition patterns of $10 \mu \mathrm{m}$ fluorescent microspheres under sine wave, wedge and staircase macrodrops. The relative surface concentration profiles are similar for different drop volumes (Fig. S5a) and match closely the relative depth profiles of the droplets (Fig. S5b). The exception is the microsphere surface concentration profile under staircase drops, which has less noticeable plateaus at higher drop volumes, likely obscured by higher variations in deposition (Fig. S5b).

The variable microparticle deposition provides an ideal method of seeding microwell arrays with desired spatial variations. Microwell arrays were fabricated according to a previous protocol $^{[32]}$ and a coated cutout (with adhesive backing) was fixed on top with centerline aligned with a row of wells (Fig. 3c). Plasma cleaning rendered the microwell array hydrophilic. Fluid was then pipetted on top of the array, filling the wells and forming a 
shaped droplet over the wells. Since the total volume of microwells in the array was small relative to the droplet volume, the droplets had virtually the same shapes as those on glass substrates. Similar to other microwell seeding methods, ${ }^{[33]}$ particles entered the microwells in two ways, initially when the well was filled with particle solution, and subsequently as sedimenting particles fell into the well from the droplet above. The particle count in each well therefore consisted of a so-called "base count", equal to the particle concentration in solution times the well volume, plus the "deposition count", equal to the volume of solution above the well times the particle concentration. The base count was constant in each well, while the deposition count was proportional to the local droplet depth. Since different particle concentrations were used in different droplets, the base count was different for each shape: approximately 50 particles for the sine shape and 40 for the wedge. The total particle counts, scaled by the maximum count, are presented in bar charts in Fig. 3c for sine wave and wedge drops, with the base count indicated by white horizontal lines. The base count was successfully removed by pre-wetting the wells with fluid not containing particles (Fig. $3 c)$. Larger variations in surface concentration were observed for the microwell seeding than for the planar surface patterning, due to the smaller areas used for counting (a single microwell vs. the larger area of a 10X microscope image) and the higher particle concentrations and corresponding aggregate formation associated with microwell seeding.

Patterning microparticles on surfaces with microscale precision in the 2D surface concentration was accomplished by wetting microscale hydrophilic regions by the method outlined in section 2.1 with fluid containing microspheres. Once microdroplets were created on the hydrophilic regions, the microspheres settled to the substrate, creating deposition patterns with microscale variations in surface concentration. Figure $3 \mathrm{~d}$ shows the deposition patterns of $1 \mu \mathrm{m}$ microspheres from micro sine wave, wedge and spiral droplets. The spatially varying surface concentrations were proportional to droplet depth (compare Figs. $2 \mathrm{~g}-\mathrm{i}$ and $3 \mathrm{~d}$ ). As with our other microdroplet experiments, $0.5 \%$ Tween 20 surfactant was used to reduce the surface tension and ensure the hydrophilic regions were fully wet. Error bars indicate the standard deviation over three repetitions. The majority of the error stemmed from the fact that the microdropet volumes were not accurately controlled with our benchtop wetting method. Similar micropatterns were observed with larger microspheres, though the lower microsphere concentrations led to higher variability in surface concentration (Fig. S5c).

Patterning cells on surfaces with microscale precision in the 2D surface concentration was achieved in a similar manner to the microsphere patterning (Fig. 4). Since the fetal bovine serum (FBS) protein found in cell media adsorbed to the OTS coating, cell media wet both the glass and the OTS-patterned regions. To resolve this issue, a small concentration, $0.1 \%$, of Pluronic F-127 surfactant was added to hinder the FBS adsorption. ${ }^{[34,35]}$ Since Pluronic sufficiently reduced the surface tension to ensure full wetting of the hydrophilic regions, other surfactants such as Tween 20 were not added. The addition of Pluronic F-127 to the cell media had a minimal effect on cell viability (Fig. S6), as noted for Pluronics in other studies. ${ }^{[34]}$ After wetting the OTS-patterned slides with cell solution, cells settled to the substrate. The resulting cell deposition patterns mirrored the microdroplet profiles. The surface concentrations along the centerlines of wedge, sine wave and spiral microdroplets had linear gradient, sine wave and angular gradient profiles, respectively (Fig. 4). Larger standard deviations in surface concentration were observed over repeated trials of patterning cells than $1 \mu \mathrm{m}$ beads. The larger variations resulted because the concentration of cells in solution was much lower than that of the $1 \mu \mathrm{m}$ beads, and the cell diameters were approximately ten times larger than that of the $1 \mu \mathrm{m}$ beads and closer to the length scale of the hydrophilic micropatterns. 


\subsection{D photolithography - shaped macrogels and microgels from shaped droplets}

A second application of shaped droplets is to pattern surfaces with hydrogels of tuned 3D geometry by crosslinking macro- or microdroplets of prepolymer solution. As an example, we have formed droplets of high concentration PEGDA 258 with photoinitiator. At the microscale, as for shaped microdroplets of water, we also added $0.01 \%$ Tween 20 surfactant to reduce the surface tension and ensure the complete wetting of our shaped hydrophilic regions. The prepolymer droplets were photocrosslinked in an oxygen free environment (Fig. 5a). At the microscale, the resulting microgels were imaged by SEM; the microgels largely retained the 3D shape of the prepolymer droplets (Fig. 5b,c). Small amounts of unreacted solution in the shallower regions were removed prior to SEM imaging. Trace amounts of oxygen in the air in the sealed Petri dish likely partially blocked the crosslinking near the air/liquid interface, which was more noticeable in shallower regions which had higher ratios of surface area to bulk volume. To prevent this in the future, a better oxygen purging system could be implemented and the inhibitor in PEGDA (4-methoxyphenol, MEHQ) could be removed by existing methods ${ }^{[36]}$ prior to UV exposure. At the macroscale, the resulting macrogels were imaged by digital camera (Fig. 5d,e). Eye-level images were digitized to obtain the centerline elevations, which compared well to FEM simulations of the prepolymer droplets (Fig. 5e). The surface tension of PEGDA 258 is 39.4 dynes $\mathrm{cm}^{-1 .[37]}$ Thus, in our simulations, we scaled all dimensions by the capillary length for PEGDA 258, $l_{c}=2.0 \mathrm{~mm}$.

\subsection{Additional remarks and future directions}

We have presented a research platform for making shaped droplets with desired 3D shapes. Benchtop techniques were employed to pattern surfaces with custom hydrophilic / hydrophobic regions and to wet the hydrophilic regions to produce shaped droplets. We have demonstrated the utility of shaped droplets for patterning microparticles and cells on surfaces with microscale precision in the surface concentration. Whether on flat substrates or in microwell arrays, the surface concentrations mimicked the droplet depth profiles. Moreover, shaped prepolymer droplets were photocrosslinked to become macro- and microgels. FEM simulations agreed well with the droplet profiles at the macro- and microscales, demonstrating that hydrophilic/hydrophobic surface patterns may be designed to produce custom microparticle surface concentrations or shaped macro- or microgels. Future research efforts should be devoted to testing different surface coatings and improving the precision of the coating and wetting methods. For example, a more sophisticated wetting method could deposit specific fluid volumes on the microscale hydrophilic regions to improve the accuracy of the microscale particle patterning and microgel synthesis.

Given the ability of shaped droplets to deposit microparticles on surfaces with controlled variations in surface concentration, a myriad of applications may be pursued. Custom 2D patterns of micro- or nanoparticles deposited by shaped droplets could be bound to the surface by existing techniques ${ }^{[18,38]}$ and used to regulate cell proliferation, attachment, and morphology. ${ }^{[23]}$ Patterns of degradable microspheres could induce custom patterns of cell migration. ${ }^{[24]}$ The morphology of colloidal particle aggregates has been shown to guide neuronal development; ${ }^{[39]}$ employing shaped droplets to deposit such colloidal particles could pattern a surface with particle aggregates with controlled variations in aggregate size and layer depth, enhancing the neuronal patterning capability. Lastly, the shaped droplet platform could pattern surfaces with controlled microscale spatial variations in bacteria concentration to study quorum sensing. ${ }^{[40]}$

While evaporation was avoided in this work, the controlled evaporation of shaped droplets could also enhance a variety of evaporative deposition techniques. The evaporative deposition and coassembly of sacrificial colloidal particles allows the synthesis of inverse- 
opal films for use in catalysis, gas adsorption, tissue engineering, and optics; ${ }^{[41]}$ employing shaped droplets to deposit colloidal particles could allow controlled 3D topography to be added to such films. The geometry of substrate surfaces can produce grid-like patterns in nanorods and nanoparticles from evaporating droplets. ${ }^{[12,13]}$ Shaped droplets could provide an additional tool for controlling the spatial deposition patterns of such nanoparticles. Square centimeter scale stacked layers of vertically aligned nanorods have been formed by the evaporation of droplets of highly concentrated solutions of nanorods; ${ }^{[7]}$ evaporating shaped droplets of such nanorod solutions could yield a 3D topography of stacked vertically aligned nanorods. A large body of literature exists on patterning colloidal micro- and nanoparticles on chemically and topographically templated surfaces ${ }^{[8-10,42]}$ Incorporating evaporating shaped droplets in such applications could provide additional spatial variations at length scales above that of the particles or particle aggregates.

Recent advances in 3D photolithography, ${ }^{[43,44]}$ soft lithography ${ }^{[14,15,45]}$ and other methods, ${ }^{[46]}$ often combined with microfluidics, ${ }^{[44,47]}$ have enabled the synthesis of tailored 3D polymer shapes. ${ }^{[48]}$ The shaped microgels fabricated in this work demonstrate the utility of shaped droplets to broaden the range of achievable 3D polymer shapes, while also providing an accurate computational design platform for such shapes. For example, the design platform presented here could help generate custom 3D droplets for existing liquid molding techniques. ${ }^{[14,15]}$ Yet another photolithography method employs patterned wetting/ non-wetting regions to direct the assembly of microgels. ${ }^{[27]}$ Shaped droplets could be incorporated with such a method to produce assembled microgel structures with enhanced 3D topography. 3D microgels have also been fabricated from chemically sol-gel transitional hydrogels by using a hydrophilic substrate and an aerosol gelling agent. ${ }^{[49]}$ Using instead a substrate patterned with hydrophilic/hydrophobic regions, shaped droplets of such hydrogels could be created and gelled without the need for molds or photolithography.

Lastly, controlled surface patterning with shaped droplets could be a tool for generating surfaces with spatially varying topography and corresponding wetting properties, such as nano- or microparticle gradients or pearl-like structures ${ }^{[50]}$ reminiscent in shape to the sine wave drops.

\section{Conclusions}

In this study we have presented benchtop techniques for patterning and wetting surfaces with custom hydrophilic / hydrophobic regions to create custom 3D droplets at the macroand microscales. Shaped droplets were used for patterning microparticles and cells on surfaces with microscale precision in the surface concentration. Shaped macro- and microgels were also synthesized and retained the 3D geometry of the prepolymer droplets. FEM simulations agreed well with the droplet shapes at the macro- and microscales, thereby providing a computational platform to design custom hydrophilic/hydrophobic regions to achieve particular drop and microgel shapes and surface patterns. Due to the vast research on particle/cell patterning and recent interest in microengineered hydrogels, we hope our shaped droplet platform will find future use in these and other active research areas.

\section{Experimental Section}

\section{Materials}

Hydrophobic WX2100 spray (Cytonix Corp., Beltsville, MD); pre-cleaned microscope glass slides (Thermo Fisher Scientific Inc., Waltham, MA); poly(ethylene glycoldiacrylate) (PEGDA, MW 258) (Monomer-Polymer \& Dajac Labs, Trevose, PA); photoinitiator (PI) 2,2-dimethoxy-2-phenyl-acetophenone (DMPA 99\%, Sigma-Aldrich Inc., St. Louis, MO); green fluorescent polymer $1 \mu \mathrm{m}$ and $10 \mu \mathrm{m}$ microspheres ( $1 \mathrm{wt} \%$ solids, Duke Scientific 
Corp., Palo Alto, CA); Polybead latex dyed violet $6 \mu \mathrm{m}$ microspheres $(2.65 \%$ Solids, Polysciences, Warrington, PA). All other reagents and tissue culture components were purchased from Sigma-Aldrich Inc. (St. Louis, MO) unless otherwise noted.

\section{Patterning glass slides with hydrophobic spray}

Hydrophilic regions were produced on glass slides with vinyl cutouts or masks (Fig. 1b). The designs used are given in Figs. S1 and S2. A few of the designs are defined by mathematical formulas and produced by a Matlab script given in SI Methods II, Table S11. Mask method: following a previous protocol, ${ }^{[20]}$ a shaped vinyl mask with adhesive backing (8300 series, MACtac, Stow, OH) was precision-cut with a Graphtec cutting plotter CE5000-60 (Graphtec America Inc., Santa Ana, CA) and fixed to a glass slide. Hydrophobic spray (WX2100) was applied and allowed to dry for 2 days, after which the mask was removed. Cutout method: shaped vinyl cutouts were precision-cut with the Graphtec cutting plotter, sprayed with WX2100 and allowed to dry. The cutouts were peeled off their backing and fixed to glass slides. Slides with custom coated hydrophobic regions (millimeter resolution) may be purchased directly from the manufacturer (e.g. Cel-Line Brand Specialty Printed Slides and Multi-Well Slides with custom coatings from Thermo Scientific's Slides and Specialty Glass division, Portsmouth, $\mathrm{NH}$ ).

\section{Patterning glass slides by microcontact printing}

Previous protocols were followed to pattern glass slides with microscale hydrophilic and hydrophobic regions. ${ }^{[27,28]}$ Glass slides were washed with dish soap and rinsed with distilled water. Polydimethylsiloxane (PDMS) stamps of the shaped microscale hydrophilic regions (as depressions into the PDMS) were fabricated according to a previous protocol ${ }^{[32]}$ (SI Methods I) and spin-coated at $1000 \mathrm{rpm}$ for $20 \mathrm{~s}$ with a $1 \%$ octadecyltrichlorosilane (OTS) solution dissolved in hexane. The stamps were then gently pressed onto the cleaned glass slides for $1 \mathrm{~min}$ (Fig. 1b). The OTS on the depressed regions did not contact the glass slide, thereby leaving uncoated hydrophilic regions on the glass slide. The dimensions of the microscale hydrophilic regions were $1 / 10^{\text {th }}$ those of the macroscopic regions (defined in Fig. S1), except for the double sine wave, whose length and width were $1 / 10^{\text {th }}$ and $1 / 5^{\text {th }}$, respectively, those of the macroscale version. Two sizes of the micro spirals were prepared, one scaled by a factor of 1/10 (appear in Figs. $2 \mathrm{~g}$ and S4d) and one scaled by 1/5 (appears in Figs. 1d, 3d, 4b,c, S4e) from the macroscale versions.

\section{Shaped droplets on macroscale hydrophilic regions}

The desired amount of fluid was pipetted onto a shaped hydrophilic region on a glass slide (Fig. 1c). The slide was tilted back and forth by 5-10 degrees to ensure full wetting. Droplets containing blue food dye were imaged by a digital camera (EOS Kiss X2, Canon USA, Inc., Lake Success, NY) with a $90 \mathrm{~mm}$ f/2.8 Di Macro Lens (Tamron USA, Inc., Commack, NY) fitted with a EM-140 DG Macro ring flash (Sigma Corp. USA, Ronkonkoma, NY) (Figs. 1c and S4a-c). Eye-level images were captured to obtain centerline depth profiles (Fig. 2a-f).

\section{Shaped droplets on microscale hydrophilic regions}

5-10 $\mu \mathrm{L}$ of the desired solution was pipetted onto a micropatterned OTS-coated glass slide, prepared as described above. The slide was then placed in a covered humid Petri dish (Fig. S3a), which was tilted at 20-30 degrees and gently tapped (Fig. S3a) to move the droplet along the patterned hydrophilic regions. As the receding edge of the drop moved along the slide, microdroplets were left on the hydrophilic regions (Fig. 1d). For water droplet characterization experiments, $0.5 \%$ Tween 20 surfactant was added to reduce surface tension and improve wetting, and in some cases $0.1 \%$ fluorescein isothiocyanate (FITC) was added 
to aid visualization. Phase contrast and fluorescence images were captured by inverted microscope (TE-2000-U, Nikon, Melville, NY) at 2X, 4X and 10X objectives (Figs. 1d and $\mathrm{S} 4 \mathrm{~d}, \mathrm{e})$. Fluorescent images were analyzed in ImageJ and Matlab to extract the centerline fluorescent intensity profiles (Fig. $2 \mathrm{~g}-\mathrm{i}$ ).

\section{Finite element method (FEM) simulations}

FEM simulations of the shapes of static droplets on the shaped hydrophilic regions were performed with Surface Evolver. ${ }^{[31]}$ Surface profiles were saved to encapsulated postscript files and processed with Adobe Acrobat and Adobe Illustrator (Figs. 1a,c,d, 3a, 5a, S4b,c). Numerical output was processed in Matlab to obtain centerline depth profiles for comparison with experiments (Figs. 2, 5d, S5b). Scripts required to generate and process all simulations in this document are supplied in SI Methods II. To compare predicted and measured surface elevations for macrodrops on slides coated by the "mask and spray" or "coated cutout" methods, only the portion of the droplet above the surface of the coating was modeled. The contact line was fixed at the top boundary of the surface coating and the simulation volume was equal to the fluid volume minus the contained volume (coating thickness times plan area of the shape, listed in Table S1 in SI Methods I). Following the simulation, the coating thickness was added to the predicted fluid elevation.

\section{Patterning surfaces with microparticles}

Microparticle surface patterning with controllable variations in the surface concentration was achieved by producing shaped droplets containing microspheres, according to the protocols above, and allowing the particles to settle (Fig. 3, S5). At the macroscale, the $6 \mu \mathrm{m}$ or $10 \mu \mathrm{m}$ microsphere solutions used for each shape were: wedge, $40 \mu \mathrm{L} 40 \mathrm{X}$ diluted microsphere solution (i.e. diluted $40 \mathrm{X}$ from stock) plus 0,35 or $85 \mu \mathrm{L}$ distilled water; sine, $20 \mu \mathrm{L} 40 \mathrm{X}$ microsphere solution plus 0 or $10 \mu \mathrm{L}$ distilled water; staircase, $40 \mu \mathrm{L} 40 \mathrm{X}$ diluted microsphere solution or $60 \mu \mathrm{L} 40 \mathrm{X}$ diluted microsphere solution plus $20 \mu \mathrm{L}$ distilled water. At the microscale, $1 \mu \mathrm{m}$ or $6 \mu \mathrm{m}$ microsphere stock solution was diluted $5 \mathrm{X}$ with water, and Tween 20 surfactant was added at a concentration of $0.1 \%$. This microsphere solution was used to wet the microscale hydrophilic regions on OTS-coated slides as described above. Surface patterns were captured by fluorescence or phase contrast microscopy. $2 \mathrm{X}$ or $4 \mathrm{X}$ images captured the overall surface pattern. For quantification, 10X, 20X and 40X objectives were used for imaging 10, 6 and $1 \mu \mathrm{m}$ microspheres, respectively. Images were captured along the centerline of a particular shape and processed in Matlab. All experiments were repeated three times.

\section{Seeding microwell arrays with prescribed concentration profiles}

Arrays of microwells (550 $\mu \mathrm{m}$ diameter, $350 \mu \mathrm{m}$ depth) spaced at $1 \mathrm{~mm}$ (center-to-center) were fabricated from PEGDA 258 with PDMS stamps according to a previous protocol. ${ }^{[32]}$ A coated cutout with adhesive backing was then fixed to the array (Fig. 3c). The centerline of the cutout was aligned with a row of microwells. Prior to droplet addition, the device was plasma cleaned to render the microwells hydrophilic. Plasma cleaning had no noticeable effect on the hydrophobic coating. Two protocols were used to seed particles. In the regular protocol, microsphere solution was pipetted directly onto empty microwells. For the wedge and sine shapes, the microsphere solution consisted of $20 \mu \mathrm{L}$ and $40 \mu \mathrm{L}$ of $20 \mathrm{X}$ diluted 10 $\mu \mathrm{m}$ microsphere solution combined with $10 \mu \mathrm{L}$ and $35 \mu \mathrm{L}$ of distilled water, respectively. In the "pre-wet" protocol, the microwells were first filled with water not containing particles; 5 $\mu \mathrm{L}$ water was spread evenly over the array. Though $5 \mu \mathrm{L}$ was $2-3$ times the total volume of the microwells within the hydrophobic boundary of the cutout $(2.2 \mu \mathrm{L}$ wedge, $1.5 \mu \mathrm{L}$ sine), excess water outside the wells evaporated quickly prior to the addition of the fluid containing microspheres. 


\section{Cell patterning by shaped droplets}

NIH-3T3 cells were cultured in Dulbecco's modified Eagle medium (DMEM, Invitrogen Corp., Carlsbad, CA) supplemented with 10\% FBS and 1\% penicillin-streptomycin (Invitrogen Corp., Carlsbad, CA) in a $5 \% \mathrm{CO}_{2}, 37{ }^{\circ} \mathrm{C}$ incubator. The cells were trypsinized for 5 mins in a $0.1 \%$ trypsin/DPBS. The cells were then stained with a solution of $0.1 \mu \mathrm{M}$ cell tracker blue CMF2 (4-chloromethyl-6,8-difluoro-7-hydroxycoumarin) (Invitrogen Corp., Carlsbad, CA) dissolved in DPBS for 15 mins. After staining, the cells were washed with DPBS and collected in DMEM with 0.1\% (w/v) Pluronic F-127 (to prevent FBS adsorption to the OTS coated surface). Droplets of this cell solution $\left(2 \times 10^{7}\right.$ cells $\left.\mathrm{mL}^{-1}\right)$ were created on the OTS-patterned slides according to the protocol above. Phase contrast and fluorescence images of the patterned cells were captured by inverted microscope (Fig. $4)$.

\section{Shaped macrogel synthesis}

The prepolymer solution consisted of $150 \mathrm{mg}$ of PI dissolved in $5 \mathrm{~mL}$ of PEGDA 258, which is in the liquid phase at room temperature. The solution was stirred at room temperature, then placed in an oven at $80^{\circ} \mathrm{C}$ for 30 mins, and then cooled to room temperature. Specific solution volumes ( $75 \mu \mathrm{L}$ wedge, $30 \mu \mathrm{L}$ sine, $60 \mu \mathrm{L}$ staircase, $30 \mu \mathrm{L}$ spiral) were pipetted onto coated glass slides to create shaped droplets, which were then placed in sealed Petri dishes filled with nitrogen gas to purge oxygen (Fig. S3b). The prepolymer drops were exposed to UV light (360-480 nm, $1.12 \mathrm{~mW} \mathrm{~cm}^{-2}$ ) in the oxygenfree environment (Figs. 5a, S3c). Different exposure times were used for different shaped macrodroplets: $1 \mathrm{~min}$ (sine); $2 \mathrm{~min}$ (wedge); $30 \mathrm{~s}$ (spiral); $1 \mathrm{~min}$ (staircase). Macrogels were imaged with digital camera in the same manner as the macrodroplets (Fig. 5c,d).

\section{Shaped microgel synthesis}

Nitrogen gas was bubbled through the PEGDA 258 prepolymer solution for 10 mins to remove dissolved oxygen. ${ }^{[51]}$ Then, $3 \% \mathrm{PI}$ and $0.01 \%$ Tween 20 were added and the solution was stirred at $80^{\circ} \mathrm{C}$ for $30 \mathrm{~min}$. Once cooled to $37^{\circ} \mathrm{C}$, the prepolymer solution was used to wet the OTS-patterned slides as described above to create shaped prepolymer microdroplets, subsequently exposed to UV light (360-480 nm; $\left.12.4 \mathrm{~mW} \mathrm{~cm}^{-2}\right)$ for $40 \mathrm{~s}$ in the oxygen-free environment (Figs. 5a, S3b,c). Any unreacted prepolymer solution was washed away by distilled water and the gels were dried in a sealed Petri dish at room temperature for 24 hours. The dried microgels were sputter coated with gold and palladium for 2 mins with a Hummer 6.2 sputter coater (Ladd Research, Williston, VT), and then imaged with SEM (JSM 5600LV, JEOL USA Inc., Peabody, MA) at an acceleration Voltage of $5 \mathrm{kV}$ and a working distance of 5-10 mm (Fig. 5b).

\section{Supplementary Material}

Refer to Web version on PubMed Central for supplementary material.

\section{Acknowledgments}

This research was funded by the NIH (HL092836, DE019024, EB012597, AR057837, DE021468, HL099073, EB008392), the Office of Naval Research, the US Army Corps of Engineers, and the National Science Foundation CAREER award (AK). HK was supported by a JSPS Fellowship for Research Abroad and Grant-in-Aid for Young Scientists (A) (23681027) from the Ministry of Education, Science and Culture, Japan. We thank Dr. Gulden Camci-Unal, Dr. Jason Nichol and Dr. Akhilesh Gaharwar for technical help and Prof. Shih-Kang Fan, Dr. Shinji Sugiura and Dr. Ben Hatton for helpful comments. 


\section{References}

1. Xia Y, Qin D, Yin Y. Curr Opin Colloid In. 2001; 6:54.Gau H, Herminghaus S, Lenz P, Lipowsky R. Science. 1999; 283:46. [PubMed: 9872735] Biebuyck HA, Whitesides GM. Langmuir. 1994; 10:2790.Darhuber AA, Troian SM, Miller SM, Wagner S. J Appl Phys. 2000; 87:7768.

2. Wier KA, Gao LC, McCarthy TJ. Langmuir. 2006; 22:4914. [PubMed: 16700573] Zhao B, Moore JS, Beebe DJ. Science. 2001; 291:1023. [PubMed: 11161212] Lam P, Wynne KJ, Wnek GE. Langmuir. 2002; 18:948. Oliveira NM, Neto AI, Song W, Mano JF. Appl Phys Express. 2010; 3:5205.Oh, CS. United States Patent. 5,904,824. 1999.

3. Hancock MJ, He J, Mano JF, Khademhosseini A. Small. 2011; 7:892. [PubMed: 21374805]

4. Delamarche E, Juncker D, Schmid H. Adv Mater. 2005; 17:2911.

5. Dupuis A, Léopoldès J, Bucknall D, Yeomans J. Appl Phys Lett. 2005; 87:024103.Brinkmann M, Lipowsky R. J Appl Phys. 2002; 92:4296.

6. Darhuber AA, Troian SM. Annu Rev Fluid Mech. 2005; 37:425.

7. Zanella M, Gomes R, Povia M, Giannini C, Zhang Y, Riskin A, Van Bael M, Hens Z, Manna L. Adv Mater. 2011; 23:2205. [PubMed: 21469223]

8. Harris DJ, Hu H, Conrad JC, Lewis JA. Phys Rev Lett. 2007; 98:148301. [PubMed: 17501317]

9. Aizenberg J, Braun PV, Wiltzius P. Phys Rev Lett. 2000; 84:2997. [PubMed: 11018995]

10. Dziomkina NV, Vancso GJ. Soft Matter. 2005; 1:265.Xia Y, Yin Y, Lu Y, McLellan J. Adv Func Mater. 2003; 13:907.

11. Yin Y, Lu Y, Gates B, Xia Y. J Am Chem Soc. 2001; 123:8718. [PubMed: 11535076]

12. Zeng H, Kristiansen K, Wang P, Bergli J, Israelachvili J. Langmuir. 2011; 27:7163. [PubMed: 21553914]

13. Hong SW, Byun M, Lin Z. Angew Chem Int Edit. 2009; 48:512.

14. Liu X, Wang Q, Qin J, Lin B. Lab Chip. 2009; 9:1200. [PubMed: 19370237]

15. Lu Y, Lin B, Qin J. Anal Chem. 2011; 83:1830.

16. Kaji H, Camci-Unal G, Langer R, Khademhosseini A. Biochim Biophys Acta. 2011; 1810:239. [PubMed: 20655984] Genzer J, Bhat RR. Langmuir. 2008; 24:2294. [PubMed: 18220435]

17. Kim SM, Lee SH, Suh KY. Lab Chip. 2008; 8:1015. [PubMed: 18584072]

18. Huwiler C, Kunzler TP, Textor M, Vörös J, Spencer ND. Langmuir. 2007; 23:5929. [PubMed: 17447799]

19. Du Y, Hancock MJ, He J, Villa-Uribe JL, Wang B, Cropek DM, Khademhosseini A. Biomaterials. 2010; 31:2686. [PubMed: 20035990]

20. Hancock MJ, Piraino F, Camci-Unal G, Rasponi M, Khademhosseini A. Biomaterials. 2011; 32:6493. [PubMed: 21684595]

21. Burdick JA, Khademhosseini A, Langer R. Langmuir. 2004; 20:5153. [PubMed: 15986641]

22. Hook AL, Voelcker NH, Thissen H. Acta Biomater. 2009; 5:2350. [PubMed: 19398391]

23. Kunzler TP, Huwiler C, Drobek T, Vörös J, Spencer ND. Biomaterials. 2007; 28:5000. [PubMed: 17720241]

24. Zhao X, Jain S, Benjamin Larman H, Gonzalez S, Irvine DJ. Biomaterials. 2005; 26:5048. [PubMed: 15769541]

25. Woodfield TBF, Blitterswijk CAV, Wijn JD, Sims TJ, Hollander AP, Riesle J. Tissue Eng. 2005; 11:1297. [PubMed: 16259586]

26. Quéré D. Ann Rev Mater Res. 2008; 38:16.1.

27. Du Y, Ghodousi M, Lo E, Vidula M, Emiroglu O, Khademhosseini A. Biotechnol Bioeng. 2010; 105:655. [PubMed: 19777588]

28. Xia Y, Mrksich M, Kim E, Whitesides GM. J Am Chem Soc. 1995; 117:9576.

29. Beysens D, Knobler CM. Phys Rev Lett. 1986; 57:1433. [PubMed: 10033448]

30. Patist A, Bhagwat S, Penfield K, Aikens P, Shah D. J Surfactants Deterg. 2000; 3:53.

31. Brakke K. Exp Math. 1992; 1:141.

32. Moeller HC, Mian MK, Shrivastava S, Chung BG, Khademhosseini A. Biomaterials. 2008; 29:752. [PubMed: 18001830] 
33. Kang L, Hancock MJ, Brigham MD, Khademhosseini A. J Biomed Mat Res A. 2010; 93:547.

34. Au SH, Kumar P, Wheeler AR. Langmuir. 2011; 27:8586. [PubMed: 21651299]

35. Amiji M, Park K. Biomaterials. 1992; 13:682. [PubMed: 1420713]

36. Dziubla TD, Lowman AM. J Biomed Mat Res A. 2004; 68A:603.

37. Jin BJ, Kim YW, Yoo JY. J Micromech Microeng. 2010; 20:035003.

38. Suzuki M, Yasukawa T, Mase Y, Oyamatsu D, Shiku H, Matsue T. Langmuir. 2004; 20:11005. [PubMed: 15568852] Bhawalkar SP, Qian J, Heiber MC, Jia L. Langmuir. 2010; 26:16662. [PubMed: 20949914] Valignat M-P, Theodoly O, Crocker JC, Russel WB, Chaikin PM. Proc Natl Acad Sci U S A. 2005; 102:4225. [PubMed: 15758072]

39. Pautot S, Wyart C, Isacoff EY. Nat Meth. 2008; 5:735.

40. Miller MB, Bassler BL. Annu Rev Microbiol. 2001; 55:165. [PubMed: 11544353] Waters CM, Bassler BL. Annu Rev Cell Dev Biol. 2005; 21:319. [PubMed: 16212498]

41. Hatton B, Mishchenko L, Davis S, Sandhage KH, Aizenberg J. Proc Natl Acad Sci USA. 2010; 107:10354. [PubMed: 20484675]

42. Smay JE, Gratson GM, Shepherd RF, Cesarano J, Lewis JA. Adv Mater. 2002; 14:1279.

43. Khademhosseini A, Langer R. Biomaterials. 2007; 28:5087. [PubMed: 17707502] Hammoudi TM, Lu H, Temenoff JS. Tissue Engineering Part C: Methods. 2010; 16:1621. [PubMed: 20412031]

44. Bong KW, Bong KT, Pregibon DC, Doyle PS. Angew Chem. 2010; 122:91.Cheung YK, Gillette BM, Zhong M, Ramcharan S, Sia SK. Lab Chip. 2007; 7:574. [PubMed: 17476375]

45. Acharya G, Shin CS, McDermott M, Mishra H, Park H, Kwon IC, Park K. J Control Release. 2010; 141:314. [PubMed: 19822178]

46. Bhaskar S, Pollock KM, Yoshida M, Lahann J. Small. 2010; 6:404. [PubMed: 19937608]

47. Dendukuri D, Doyle PS. Adv Mater. 2009; 21:4071.

48. Oh JK, Drumright R, Siegwart DJ, Matyjaszewski K. Progress in Polymer Science. 2008; 33:448.Khetan S, Burdick JA. Soft Matter. 2011; 7:830.

49. Lee W, Son J, Yoo S-S, Park J-K. Biomacromolecules. 2010; 12:14. [PubMed: 21138286]

50. Wu S-Z, Wu D, Yao J, Chen Q-D, Wang J-N, Niu L-G, Fang H-H, Sun H-B. Langmuir. 2010; 26:12012. [PubMed: 20499864]

51. Dziubla TD, Torjman MC, Joseph JI, Murphy-Tatum M, Lowman AM. Biomaterials. 2001; 22:2893. Received: ((will be filled in by the editorial staff)) Revised: ((will be filled in by the editorial staff)) Published online on ((will be filled in by the editorial staff)). [PubMed: 11561895] 

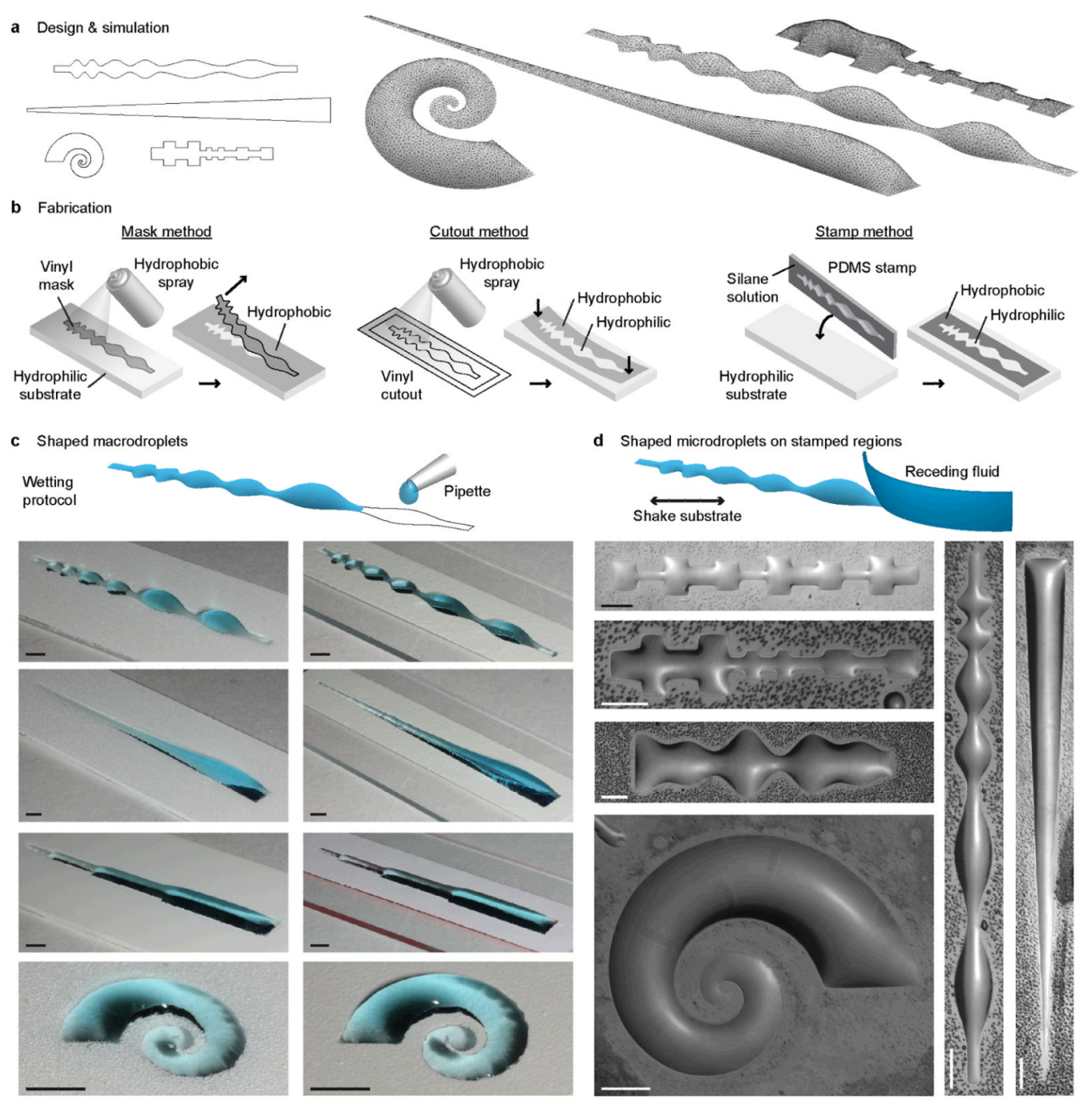

Figure 1.

Design and fabrication of shaped hydrophilic regions to produce shaped droplets. (a) Design and simulation. The shapes of droplets on different hydrophilic regions (at left) were calculated by Surface Evolver. (b) Coated slide fabrication by mask, cutout, and stamp methods. In the mask method, a shaped vinyl mask was fixed on a glass slide. Hydrophobic spray was applied and allowed to dry. The mask was removed. In the cutout method, vinyl sheets were cut with plotting cutter. Hydrophobic spray was applied and allowed to dry. The coated cutout was then peeled from its backing and affixed to a glass slide. In the stamp method, a PDMS stamp was spin-coated with silane solution and then pressed onto a glass slide. The solution was allowed to dry, forming a hydrophobic boundary enclosing a hydrophilic region. (c) Macrodroplets formed by pipetting fluid onto sine wave, wedge, staircase and spiral hydrophilic regions created by mask (first column) and cutout method (second column). Scale bars $3 \mathrm{~mm}$. (d) Microdroplets formed by gently shaking substrate to deposit fluid on different hydrophilic regions prepared by the stamp method. Scale bars 300 $\mu \mathrm{m}$. 

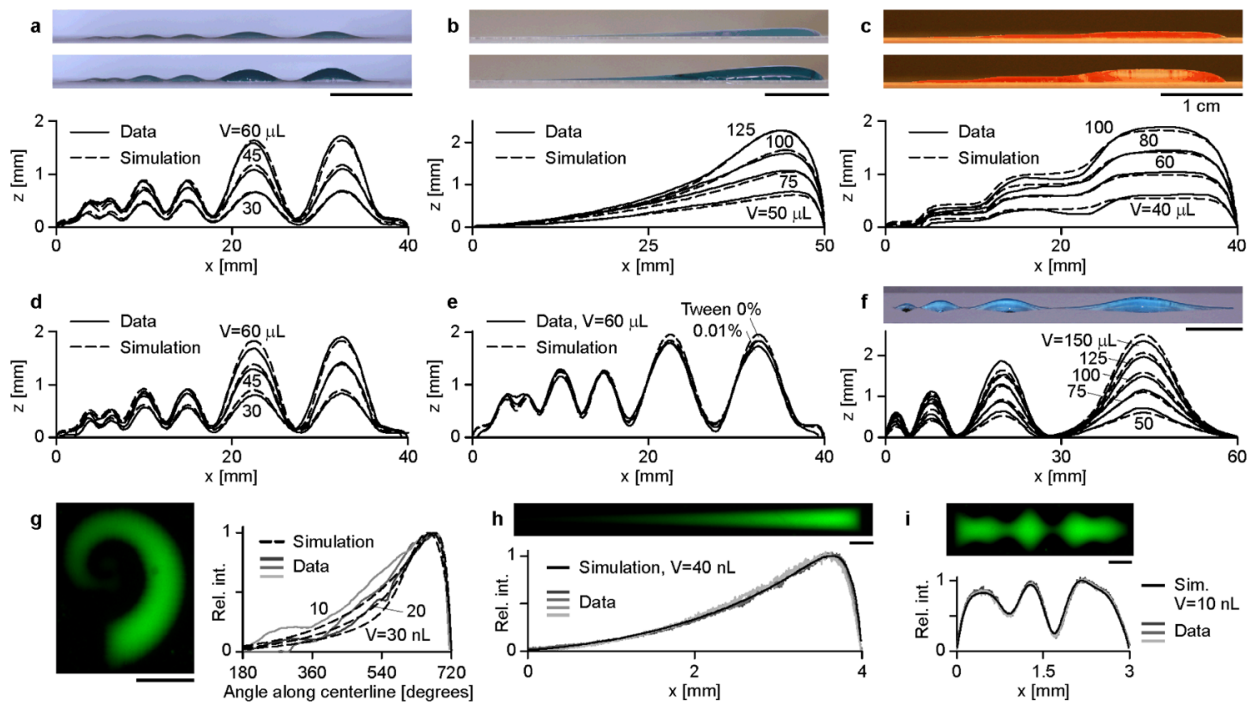

Figure 2.

Centerline elevation profiles of shaped droplets and dependence on volume, surface tension, length scale and coating type. Macro (a) wedge, (b) sine wave, and (c) staircase on cutout devices, for different volumes of distilled water. (d) Macro sine waves on slide coated by mask method. (e) Macro sine waves with and without surfactant. (f) Macro multi-wedge droplet tests wavelength and slope effect of hydrophilic wedge region. (g-i) Microdroplets with $0.5 \%$ Tween 20 . Fluorescence images quantified by plots of relative intensity for micro (g) spiral, (h) wedge, and (i) sine wave. Scale bars (a-f) $1 \mathrm{~cm}$ and (g-i) $400 \mu \mathrm{m}$. In (a-i), Surface Evolver simulations plotted for comparison. 

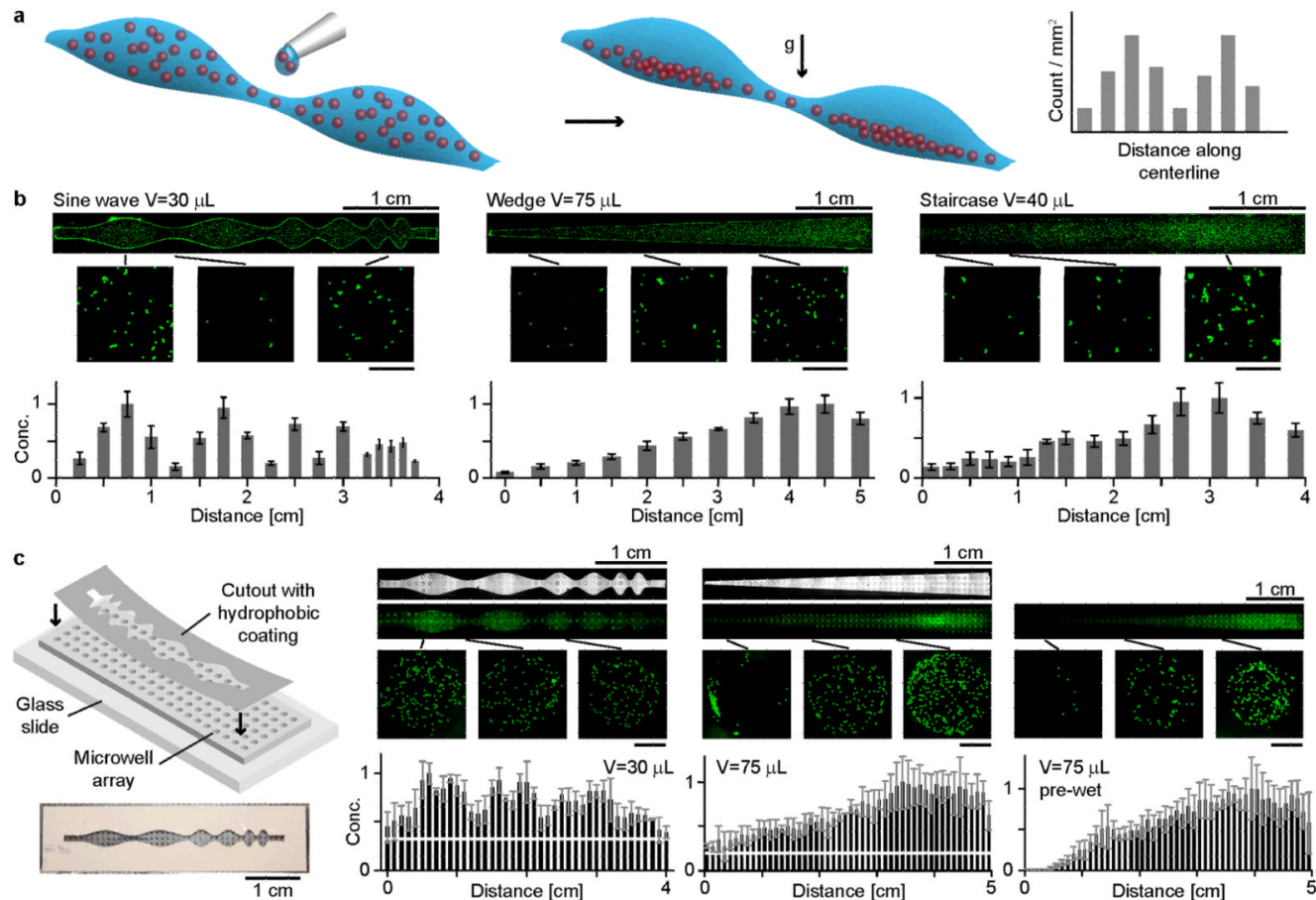

Distance $[\mathrm{cm}]$
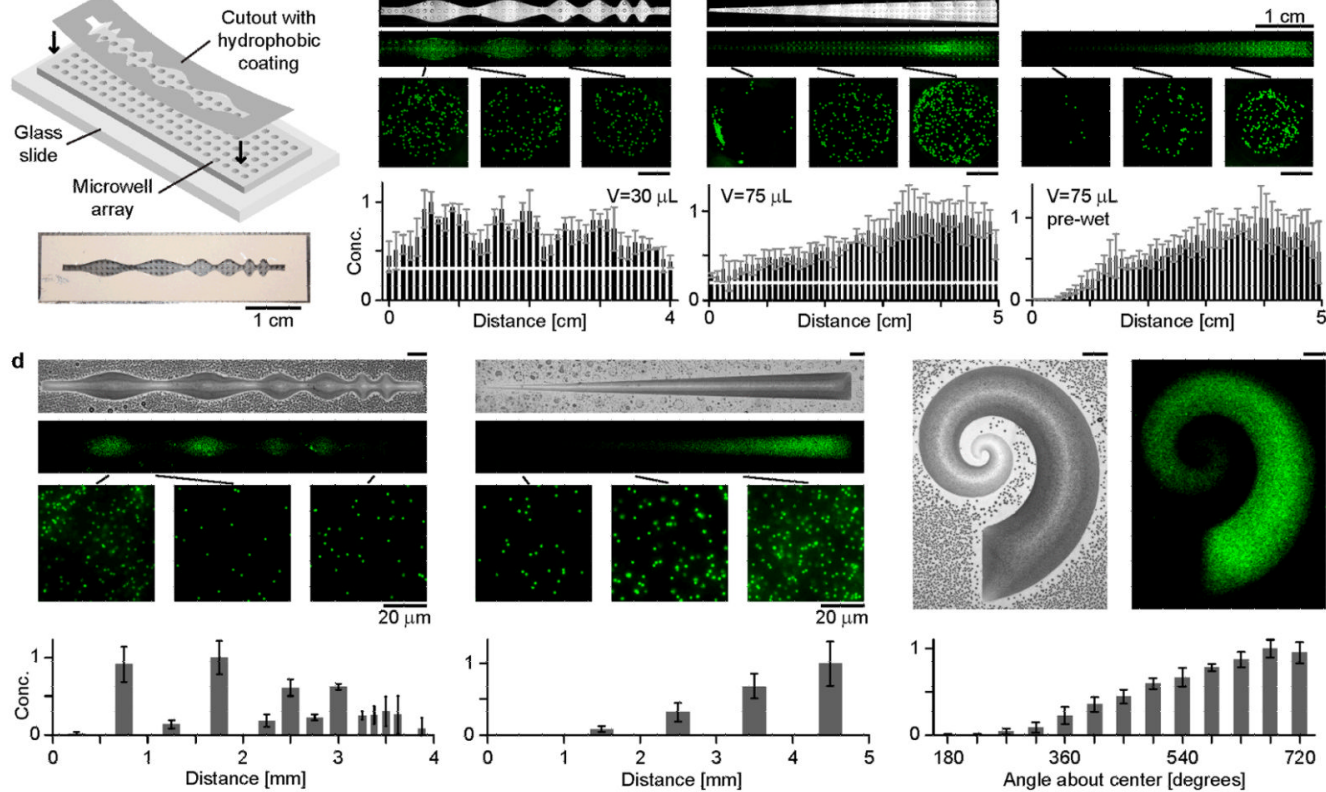

Figure 3.

Microparticle patterning by gravitational settling within shaped droplets. (a) Schematic. Drop fluid with particles is deposited on shaped hydrophilic region. Particles settle under gravity. Higher surface concentrations occur under deeper regions of the drop. (b) Patterning of $10 \mu \mathrm{m}$ microspheres within sine wave, wedge and staircase macrodrops. Stitched $2 \mathrm{X}$ fluorescence microscope images demonstrate overall surface concentration profile, while sub-images of 10X zooms and bar charts quantify surface concentration near centerline. (c) Seeding microwell arrays with $10 \mu \mathrm{m}$ microspheres. Sine wave and linear gradient concentration profiles are obtained from sine wave and wedge droplets. Stitched phase images demonstrate device layout. Fluorescent images and bar charts interpreted as in (b), except zooms and each bar in charts represent a single microwell. Horizontal white lines in bar charts indicate base count, successfully removed by pre-wetting wells with pure fluid (right). (d) Patterning of $1 \mu \mathrm{m}$ microspheres on substrates under sine wave, wedge and spiral microdroplets. Matching stitched $4 \mathrm{X}$ phase and fluorescent images illustrate droplet and overall surface concentration, while 40X zooms and bar charts indicate surface concentration near centerline. Distance along spiral centerline measured in degrees about center, where 720 degrees corresponds to the large end. In (b-c), scale bars $200 \mu \mathrm{m}$, unless noted. Error bars denote the standard deviation over three repetitions. 

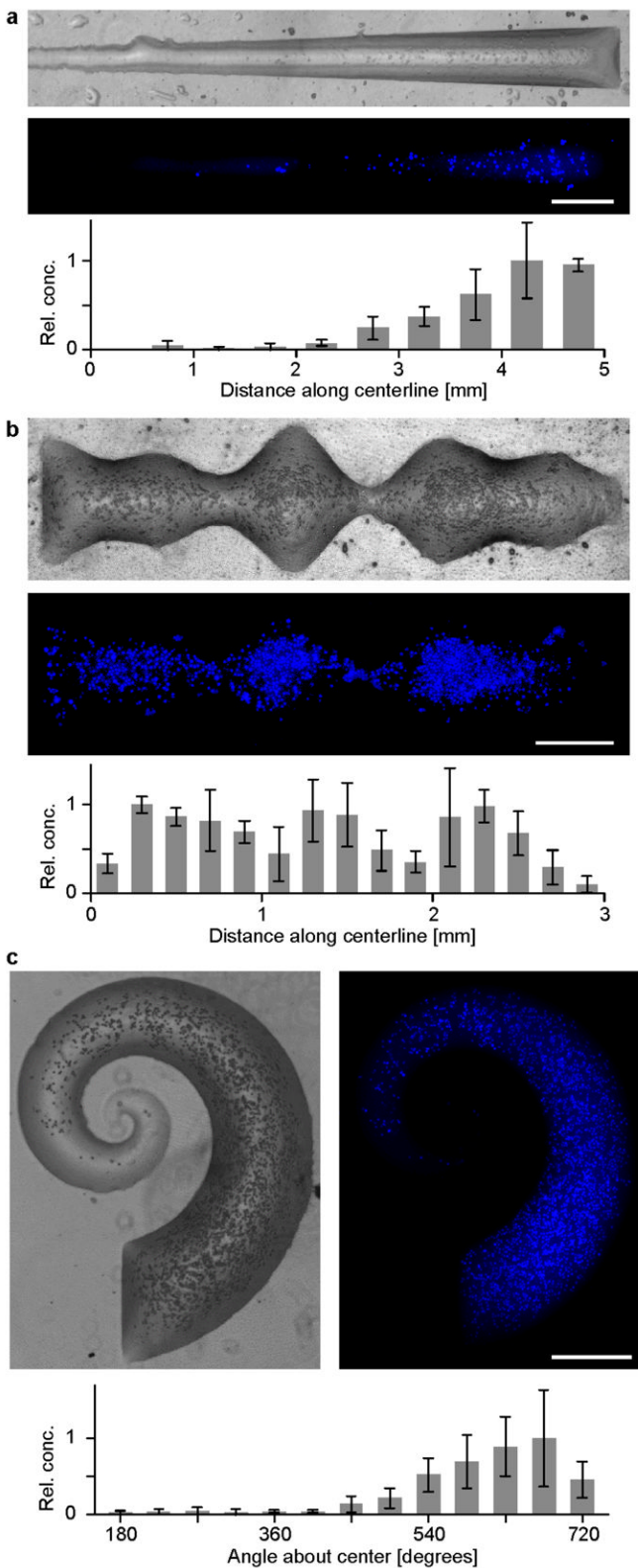

Figure 4.

Cell patterning by gravitational settling within shaped microdroplets. NIH-3T3 cells settled on the bottom of (a) wedge, (b) sine wave and (c) spiral microdroplets of cell media on OTS-patterned surfaces. Cells were stained with cell tracker blue to facilitate visualization. Bar charts quantify the relative surface concentration of cells near the droplet centerlines. Error bars denote the standard deviation over three repetitions. In (c), distance along spiral centerline measured in degrees about its center, where 720 degrees corresponds to the large end. Scale bars $400 \mu \mathrm{m}$. 


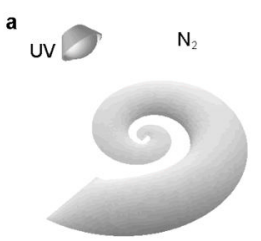

b

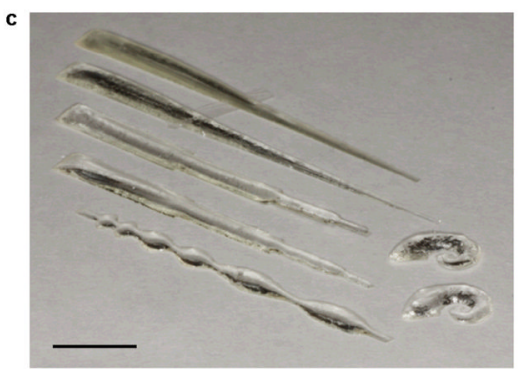

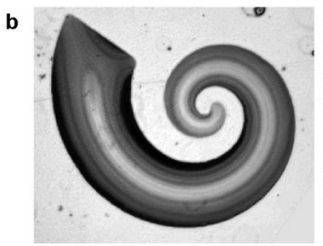
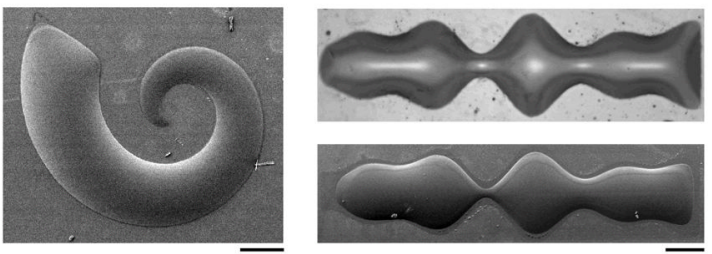

d

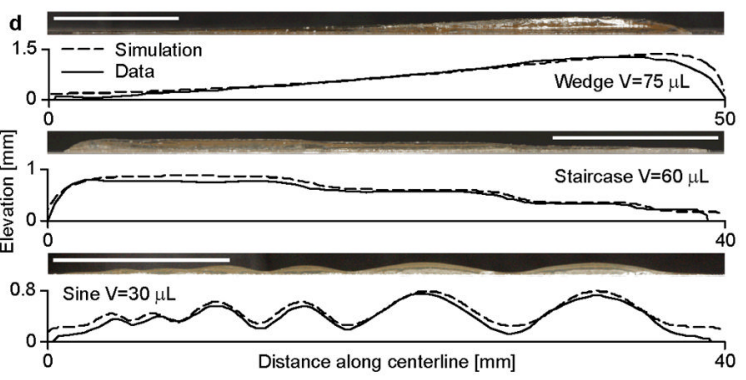

Figure 5.

Shaped micro- and macrogels. (a) Photocrosslinking protocol. Shaped droplet of prepolymer solution exposed to ultraviolet light in an oxygen-free environment. (b) Phase contrast microscope image of spiral microdroplet before crosslinking (left) and SEM image of microgel after crosslinking (right). Similarly, double sine wave microdroplet before crosslinking (top) and microgel after crosslinking (bottom). Scale bars $300 \mu \mathrm{m}$. (c) Shaped macrogels. Clockwise from bottom left: sine wave, staircase (2), wedge (2), and spiral (2) macrogels. Scale bar $1 \mathrm{~cm}$. (d) Comparison of wedge, staircase and sine wave macrogel centerline elevation profiles with theoretical droplet profiles. Scale bars $1 \mathrm{~cm}$. 